\title{
Research on Inventory and Distribution Collaborative Optimization of Building Materials Supply Chain
}

\author{
Shuai Ma ${ }^{1, a}$, Xifu Wang 1, b, Yajun Liu ${ }^{1, c}$ and Ze Gao ${ }^{2, d}$ \\ ${ }^{1}$ School of Traffic and Transportation, Beijing Jiao tong University, Beijing 100000, China \\ ${ }^{2}$ Beijing far - Communications Technology Co., Ltd, Beijing 100000, China \\ a16125754@ bjtu.edu.cn, bxfwang1@bjtu.edu.cn, ${ }^{c} 14125764 @ b j t u . e d u,{ }^{d}$ rebacca_yj@163.com
}

Keywords: Virtual Inventory, Inventory and Distribution, Collaborative Optimization, Allocation, Genetic Algorithm.

\begin{abstract}
In this paper, we discuss about the three logistics systems which consist of one manufacturer, one dealer and many customers. It is based on the particularity of the construction material consumption being slowly, we study the inventory and distribution coordination optimization problem based on the virtual inventory management. First of all, under the premise of analyzing the cost constitution of building material supply chain, we build a collaborative optimization model of inventory and distribution based on the minimum cost of the whole supply chain, and solve it by genetic algorithm. At last, by simulating the example whit Mat lab Software, we finish verifying the correctness and validity of the model, and get the minimum cost of the supply chain and the corresponding allocation scheme.
\end{abstract}

\section{Introduction}

At present, research on supply chain synergies has been progressed by leaps and bounds. But, research on the supply chain of building materials is relatively few. Building materials supply chain costs remain high, it mainly depends on the special nature of the building materials industry products, building materials is too bulky. This paper is based on the idea of virtual inventory management, the methods on traditional virtual inventory management do a certain extension and discusses the allocation of goods in customers in the supply chain of building materials. Aim at providing useful ideas and methods for enterprises to reduce logistics costs and improve management level.

\section{Model Construction}

This chapter will establish a collaborative inventory and distribution optimization model based on virtual inventory and provide the basis for the information sharing center's decision-making.

\subsection{Conditional Assumptions}

In order to facilitate the establishment of the model and to achieve good results, the following assumptions are made here:

(1) After the redeployment, the allocation of goods out of the customer cannot be out of stock situation in the follow-up production activities. (2) The location of the manufacturer, dealer, customer is known and fixed.(3) The information sharing center only provides information services, no inventory; The manufacturer does not have stock.(4) In this paper, the model discuss the cost to be optimized. (5) In this paper, all of the customers discussed are supplied by the same manufacturer and focus on the allocation among customers, in order to facilitate the calculation of time. (6) In order to facilitate the calculation of time, the unified replenishment of all out-of-stock customers, the manufacturer in the replenishment and the completion of the production of all the shortages. (7) Due to the variety of building materials, in the project, its dosage, price and other parameters are also different. Take a building material as an example in the paper.

\subsection{Symbol Definition}

$X_{i j}-\mathrm{j}$ state identification, identify the transfer of customer I to customer $\mathrm{j}$. if customer I has to allocate to customer $\mathrm{j}, X_{i j}=1$; if not $X_{i j}=0 . \mathrm{Qij} —$ the amount of allocation of customer I to customer 
j. $Q_{i}$ — the replenishment of the producer to the customer I. $d_{i j}$ - the distance between customer I and customer j. $S_{i}$ - the distance between the manufacturer and customer I. $L_{i}$ - the current stock of customer I. $N_{i}$ — customer I out of the total building materials. $N_{D i}$ the minimum demand for customer I during replenishment. $N_{T i}$-maximum allowable amount for client i. $N_{i j}$ - the number of customers I are transferred to customer j. $V_{c i}$ customer I consumption of building materials speed, $\mathrm{kg} /$ day. $V_{p}$ — the production rate of building materials, $\mathrm{kg} / \mathrm{day} . V_{T}$ transport speed of building materials, $\mathrm{km} / \mathrm{h}$. Ti_customer I project delay time. ti— the time takes for the replenishment to reach customer i.CK_ - the cost of a single order. CY — unit of goods, transport costs per unit distance, $Y / \mathrm{kg} \bullet \mathrm{km}$.TC_— total cost of supply chain. $Q_{H i}$ — the number of workers in the project customer I. $Q_{M i} \longrightarrow$ the number of machines in the project customer i.PH— — the unit price of labor cost in the project, $¥ /$ day. Ps— — unit price of materials in the project, $¥ / \mathrm{kg} . P_{M i}-$ unit price of mechanical work in the project customer I, Y/tai classes. $P_{A i}$ — the unit price of the management fee in the project customer I, Y/ day.PR_—allocating the goods out of the customer rebate unit price in the entire system, $Y / \mathrm{kg}$. Z—-transport loss rate of materials

\subsection{Model Construction}

Apply the symbols defined in the previous section, it establish supply chain inventory and build materials synergistic optimization mathematical model. The cost per part is calculated one by one, prepare for the establishment of the model.

The order cost, transportation costs, out of stock costs, rebate costs, the cost of goods added together:

$$
\begin{aligned}
& T C=C_{1}+C_{2}+C_{3}+C_{4}+C_{5} \\
& =\mathrm{n} \times C K+\sum_{i=1}^{N} \sum_{j=1}^{N}\left(X_{i j} \times Q_{i j} \times d_{i j} \times C Y+X_{i j} \times P_{R} \times Q_{\mathrm{ij}}\right) \\
& +\sum_{i=1}^{N}\left(\left(N_{i}-\sum_{j=1}^{N} Q_{j i}\right) \times S_{i} \times C Y+\left(57 \times Q_{\text {li }}+P_{\text {li }} \times 50 \% \times Q_{\text {li }}+P_{A \mathrm{i}}\right) \times\left(\frac{\sum_{i=1}^{N} N_{i}}{V_{P}}+\frac{S_{i}}{V_{T}}-\frac{\sum_{j=1}^{N} Q_{j i}}{V_{C i}}\right)\right) \\
& +\left(P_{S} \times\left(\sum_{i=1}^{N} N_{i}+\sum_{i=1}^{N} \sum_{j=1}^{N} Q_{i j}\right)+\sum_{i=1}^{N} \sum_{j=1}^{N}\left(X_{i j} \times Q_{i j} \times d_{i j} \times C Y\right)+\sum_{i=1}^{N}\left(Q^{\prime}{ }_{i} \times S_{i} \times C Y\right)\right) \times Z \\
& \text { S.t. } \sum_{i=1}^{N} N_{i}=\sum_{i=1}^{N} Q^{\prime}{ }_{i} \cdot \sum_{j=1}^{N} Q_{i j} \leq N_{T i} \cdot \sum_{j=1}^{N} Q_{i j} \leq N_{j} . Q^{\prime}{ }_{i} \leq N_{i} \cdot Q_{i j} \geq 0 .
\end{aligned}
$$

Assumptions: a class for a mechanical continuous work 8 hours, the cost of a class equipment to take 1,500 yuan. Management fee of 10,000 Yuan per day. Rebate priced at 100 yuan per kilogram of goods reward.

\section{Algorithm Design}

This chapter uses the idea of genetic algorithm to solve the model, calculate the cost of the entire supply chain to minimize the transfer program

\subsection{Chromosome Encoding}

In the collaborative optimization model, focusing on the quantity of goods Qij that customer $\mathrm{i}$ transfers to customer $\mathrm{j}$. The above constraints are used as constraints, and make the following addition.

(1) $\mathrm{I}=\mathrm{j}$, indicates the amount of transfer from client $\mathrm{i}$ to itself, at this time $\mathrm{Qij}=0$;

(2) The amount of client $i$ to customer $\mathrm{j}$ is $\mathrm{Y}, \mathrm{Qij}=\mathrm{Y}, \mathrm{Qij}=0$;

In order to facilitate the calculation, this paper does not use the transmission scheme as a chromosome, the call sequence is selected.

\subsection{Set the Initial Population}

According to the first step, it defines the method and construct the initial population.

\subsection{Fitness Function}

The higher the fitness, the probability of inheritance to the next generation is greater. 


\subsection{Select Operator}

If Population number are $\mathrm{M}$ and the fitness of the individual $\mathrm{I}$ is fi, the probability of an individual being selected is: $\mathrm{P}=f_{i} / \sum_{k=1}^{M} f k$.

\subsection{Crossover Operator}

The crossover operator in this paper adopts a single point crossover operator, as shown in figure 1 .

\begin{tabular}{|c|c|c|c|c|c|c|}
\hline The patent 1 & 110 & 11 & $\begin{array}{l}\text { Single point } \\
\text { crossover }\end{array}$ & 110 & 00 & Sub-individual1 \\
\hline The patent 2 & 011 & 00 & & 011 & 11 & Sub-individual2 \\
\hline
\end{tabular}

\subsection{Mutation Operator}

Fig. 1 Schematic Diagram of Single Point Intersection

The mutation operation in this paper uses a basic bit mutation operator or a uniform mutation operator.

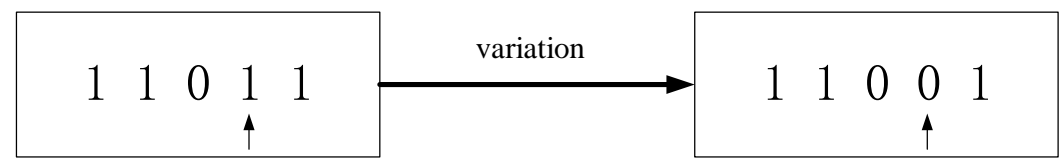

Fig. 2 Schematic Diagram of Variation Operation

\subsection{Reinsert}

In accordance with the rules, it is re-inserted into the higher fitness of the fathers, replaces the lower fitness of the parents in the individual and achieves the purpose of optimization, so that a new parent with higher fitness enters into the inheritance of the next generation.

\subsection{Termination Condition}

In this paper, the number of genetic evolution is as the end of the conditions. When the number of evolution is reached, the individual with the highest fitness in the population is the optimal solution.

\subsection{Other Parameters}

$\mathrm{M}$ is population size, generally taken as 20 100; $\mathrm{Pc}$ is crossover probability, generally taken as0.4 0.99; Pm is mutation probability, generally taken as 0.0001 0.1; GGAP is generation gap, generally taken as $0.5 \sim 1$.

\section{Example Design And Analysis}

This chapter will be based on the previous chapters, Collaborative Optimization Model and Algorithm for Inventory and Distribution of Building Materials Supply Chain do simulation calculation through an example, verify its correctness and analyze the results.

\subsection{Example Background}

Shandong Province, a regional building materials supply chain is as the background, which is located in the JN City Economic Development Zone. We select 20 customers under construction in this zone. Due to some objective reasons, 8 customers of building materials are out of stock, others are still margin of building materials.

\subsection{Data Collation}

The production rate of building materials is $\mathrm{VI}=1000 \mathrm{Kg} /$ day, transportation speed is $\mathrm{V} 3=50 \mathrm{Km}$ / h. Each customer's building materials consumption rate is respectively 150,100,200,100,240, 190, 140, 240, 390, 200, 110, 200, 330, 220, 670, 320, 330, 530, 230, 640. Unit: (Kg / day).The manufacturer, supplier, customer location has been given, it is 129, 117, 72, 118, 60, 119, 131, 147, 133, 138, 107, 142, 60, 59, 120, 63, 113, 148, 82, 107.

\subsection{Calculation Process and Result Analysis}

(1) Based on the above information, we find the customer's replenishment time and the minimum demand amount of each customer is obtained: 1350, 900, 1800, 900, 1920, 1710, 1260, 2160, 3510, $1800,990,1800,2640,1760,6030,2560,2970,4770,2070,5760$ (unit: km), The maximum transfer 
amounts are respectively 150,300, 200, 800, 1480, 590, 840, 1280, 690, 2200, 1010, 1700, 0, 0, 0, 0, $0,0,0,0$ (Unit: $\mathrm{kg}$ ) . In this paper, a genetic algorithm is used in the study uses Mat lab programming to solve the algorithm. It gets $¥ 5836138$ after 50 iterations.

(2) In the paper, if we do not use the transfer program, we can calculate the total cost of the supply chain according to the cost model in Chapter II. Customers 13 to 20 the amount of demand are respectively $4000,2800,8800,18000,5000,17500,4400,17800$,(unit:kg); The distance to the supplier is $60,59,120,63,113,148,82,107$, (unit: $\mathrm{km}$ ); transport unit price is 0.5 yuan $/ \mathrm{kg} * \mathrm{~km}$.

(3) Out of stock cost: the replenishment times for customers 13 through 20 are respectively 8,8 , $9,8,9,9,9,9$, (unit :day); the number of participants in the project is $388,203,256,215,334,326$, 219, 334,(unit: people). The number of participating machines is15, 14, 11, 13, 17, 16, 13, 14, (unit: Tai).

The total cost of the supply chain: 6662137.

The total cost of the allocation scheme is $¥ 5,836,138$ compared to the total cost of the order scheme 6662137, which verifies its correctness and validity, it can reduce the cost of building materials supply chain by $12 \%$.

\section{Conclusion}

The genetic algorithm is chosen as the algorithm of the model, the solution of the model and the specific process are determined. And it design each step. The simulation process is simulated by Mat lab code and solved by a practical example. By comparison with the ordering situation, it verify the effectiveness of collaborative inventory and distribution optimization.

\section{References}

[1]. Tiaras .G. Pooling in multi-location periodic inventory distribution system [J]. Omega, Int. JMg mt. Sci, 1999, 27 (I):39-59.

[2]. N.C. Sim PS On. Central versus local multiple stage inventory planning: An analysis of solution s [J]. European Journal of operational Research.2007. (181):127-138.

[3]. William J. O'Brien, Kerry London, Ruben Vrijhoef. Construction supply chain modelling: A re search review and interdisciplinary research agenda [J]. Proceedings IGLC-10, Gramado, Brazi 1, 2002: 129-148.

[4]. Landers T L, Cole M H, Walker B, et al. The virtual warehousing concept [J]. Transportation $\mathrm{R}$ esearch Part E, 2000, 36(2):115-125.

[5]. Lintao Liu, Optimal Design of Logistics Warehouse Management System Based on Three - dim ensional Service Platform [J]. Science \& Technology., 2014, 22(2):176-181. 